\title{
Clinical and pathological analysis of benign brain tumors resected after Gamma Knife surgery
}

\author{
Clinical article
}

\author{
Ali Liu, M.D., ${ }^{1}$ Jun-Mei Wang, M.D., ${ }^{2}$ Gui-Lin Li, M.D., Ph.D., ${ }^{2}$ Yi-Lin Sun, M.D., ${ }^{2}$ \\ Shi-Bin Sun, M.D., ${ }^{1}$ Bin Luo, M.D., ${ }^{1}$ ANd Mei-Hua Wang, M.D. ${ }^{1}$ \\ ${ }^{1}$ Gamma Knife Center and ${ }^{2}$ Department of Pathology, Beijing Neurosurgical Institute, Beijing Tiantan \\ Hospital, Capital University of Medical Sciences, Beijing, China
}

\begin{abstract}
Object. The goal of this study was to assess the clinical and pathological features of benign brain tumors that had been treated with Gamma Knife surgery (GKS) followed by resection.

Methods. In this retrospective chart review, the authors identified 61 patients with intracranial benign tumors who had undergone neurosurgical intervention after GKS. Of these 61 patients, 27 were male and 34 were female; mean age was 49.1 years (range 19-73 years). There were 24 meningiomas, 18 schwannomas, 14 pituitary adenomas, 3 hemangioblastomas, and 2 craniopharyngiomas. The interval between GKS and craniotomy was 2-168 months, with a median of 24 months; for 7 patients, the interval was 10 years or longer. For 21 patients, a craniotomy was performed before and after GKS; in 9 patients, pathological specimens were obtained before and after GKS. A total of 29 patients underwent GKS at the Beijing Tiantan Hospital. All specimens obtained by surgical intervention underwent histopathological examination.

Results. Most patients underwent craniotomy because of tumor recurrence and/or exacerbation of clinical signs and symptoms. Neuroimaging analyses indicated tumor growth in 42 patients, hydrocephalus in 10 patients with vestibular schwannoma, cystic formation with mass effect in 7 patients, and tumor hemorrhage in 13 patients, of whom 10 had pituitary adenoma. Pathological examination demonstrated that, regardless of the type of tumor, GKS mainly induced coagulative necrosis of tumor parenchyma and stroma with some apoptosis and, ultimately, scar formation. In addition, irradiation induced vasculature stenosis and occlusion and tumor degeneration as a result of reduced blood supply. GKS-induced vasculature reaction was rarely observed in patients with pituitary adenoma. Pathological analysis of tumor specimens obtained before and after GKS did not indicate increased tumor proliferation after GKS.

Conclusions. Radiosurgery is effective for intracranial benign tumors of small size and deep location and for tumor recurrence after surgical intervention; it is not effective for intracranial tumors with symptomatic mass effect. The radiobiological effects of stereotactic radiosurgery on the benign tumors are mainly caused by cellular and vascular mechanisms. Among the patients in this study, high-dose irradiation did not increase tumor proliferation. GKS can induce primary and secondary effects in tumors, which could last more than 10 years, thereby warranting long-term follow-up after GKS.
\end{abstract}

(http://thejns.org/doi/abs/10.3171/2014.8.GKS141439)

\section{KeY WORDS • Gamma Knife surgery stereotactic radiosurgery}

histopathology $\quad$ brain benign tumors

$\mathrm{G}$ AMma Knife surgery (GKS) was introduced for the treatment of intracranial tumors almost half a century ago. It is estimated that by the end of 2012 , more than 260,000 cases of benign intracranial tumors had been treated with GKS at an annual rate of more than 20,000 cumulative cases around the world. However, the pathological effects of high-dose focused gamma irradiation on benign brain tumors and the pathophysiological consequences are unclear. Since its establishment in 1994, our institution (Gamma Knife Center, Beijing Neurosurgical Institute, Beijing Tiantan Hospital)

Abbreviations used in this paper: GKS = Gamma Knife surgery; $\mathrm{SRS}=$ stereotactic radiosurgery. has treated more than 9000 cases of benign brain tumor, accounting for $53.3 \%$ of all patients. In the study reported here, we identified 61 patients from our hospital with a benign brain tumor for which GKS had failed and was later resected. We evaluated the long-term pathological changes in benign brain tumors after GKS.

\section{Methods}

\section{Patient Population}

From April 1996 through January 2013, a total of 61 benign brain tumors had been treated with GKS (29 at our hospital and 32 elsewhere) and were subsequently re- 
A. Liu et al.

sected at our hospital. There were 24 meningiomas, 18 schwannomas, 14 pituitary adenomas, 3 hemangioblastomas, and 2 craniopharyngiomas (Table 1). Our study cohort consisted of 27 male and 34 female patients, 18-78 years of age (mean age 49.1 years). The interval between GKS and resection was 2-168 months (mean 41.9 months, median 24 months); for 7 patients, the interval was more than 10 years. A total of $21(34.4 \%)$ patients in this group had undergone surgical intervention before GKS. This study was approved by our institutional review board. Patients' records were retrospectively reviewed and the data were not used to guide treatment decisions.

Among the 24 meningiomas, 6 were petroclival, 5 were cavernous sinus, 4 were sphenoid ridge, 2 were cerebellopontine angle, 2 were tentorium, 2 were frontal and parietal lobes, 2 were posterior third ventricle, and 1 was intracerebroventricular. Among the 18 schwannomas, 14 were acoustic, 3 were trigeminal, and 1 was vagal. Among the 14 pituitary adenomas, 12 were nonfunctional pituitary adenomas, 1 was a prolactinoma, and 1 was a mixed pituitary adenoma. All 3 hemangioblastomas were located in the cerebellum, and the 2 craniopharyngiomas were suprasellar.

\section{GKS Treatment}

Of the 61 patients, 29 underwent radiosurgery with Leksell Gamma Knife models B, C, or Perfexion (Elekta AB) at our institution from December 1994 through May 2002, June 2002 through September 2011, or since October 2011, respectively. Among these 29 patients, 10 had meningioma, 9 schwannoma, 9 pituitary adenoma, and 1 craniopharyngioma. Tumor volumes were 1.6-26.0 $\mathrm{cm}^{3}$ (mean $8.528 \mathrm{~cm}^{3}$, median $6.15 \mathrm{~cm}^{3}$ ). The prescription dose to the tumor margin was 8-18 Gy (mean 12.7 Gy, median $13 \mathrm{~Gy}$ ), with an isodose curve of 40\%-60\% (mean 44.6\%). The other 32 patients underwent GKS at other hospitals, which used different equipment and did not have complete treatment information.

\section{Neuroimaging}

All 61 patients underwent MRI and/or CT (including unenhanced and contrast-enhanced scans) before and after GKS and before final resection.

\section{Tumor Resection and Pathology}

All 61 patients underwent final craniectomy at our hospital, and tumor specimens were collected from all patients. Tumor specimens were fixed in $10 \%$ formalin, processed for paraffin-embedding, cut into $5-\mu \mathrm{m}$ sections, and stained with $\mathrm{H} \& \mathrm{E}$. Sections were further processed for immunohistochemistry of vimentin, epithelial membrane antigen, S100 protein, glial fibrillary acidic protein, synaptophysin, chromogranin A, CD34 class II, cytokeratin 8 and 18 , keratin 8 , keratin 5 and 6 , and carcinoembryonic antigen for diagnosis of neuroepithelial tumors, hemangiopericytomas, and metastases. Nine specimens were processed for electron microscopy. For an additional 9 patients, tumor specimens were collected before and after GKS: 4 meningiomas, 2 schwannomas, 2 pituitary adenomas, and 1 craniopharyngioma. The effect

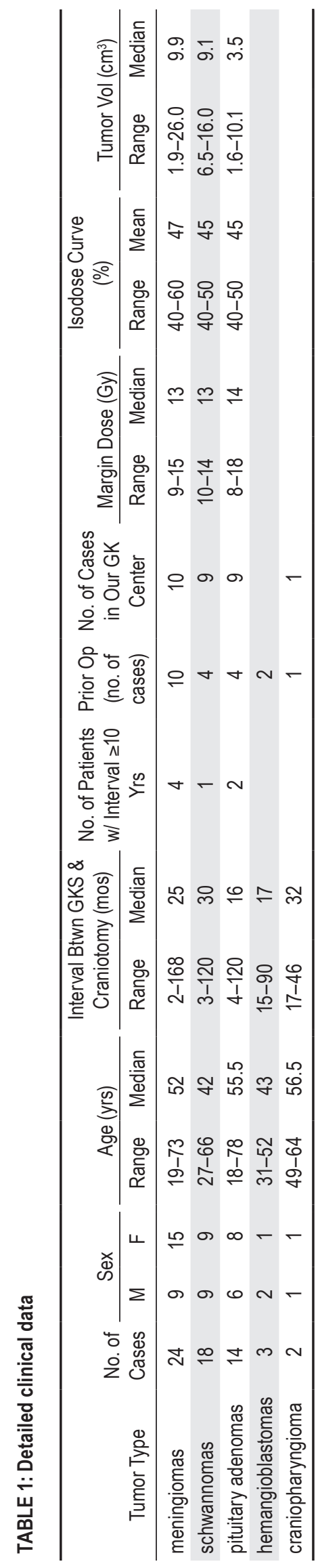


of gamma radiation on tumor proliferation was evaluated by comparing the neuropathology of specimens before and after GKS.

\section{Results}

\section{Clinical Characteristics}

Most patients had undergone tumor resection after GKS because of worsening of tumor-related signs and symptoms. Neuroimages indicated enlargement of $41 \mathrm{tu}-$ mors (19 of which had been larger than $10 \mathrm{~cm}^{3}$ before GKS), shrinkage of 4 tumors, hemorrhage for 13 tumors (10 of which were pituitary adenomas), and cyst formation for 3 tumors. In this group, hydrocephalus was found in 10 patients with acoustic schwannoma. Peritumoral brain edema occurred in 10 patients.

\section{Pathological Findings}

The interval between GKS and resection was 2-168 months (median 24 months) (Table 2). Radiation reaction developed in all tumor specimens at several months after GKS, evidenced by homogeneous coagulation necrosis with scattered cell debris and apoptotic cells. A clear boundary was observed in areas of radiation necrosis (Fig. 1). At several years after GKS, coagulation necrosis became even more extensive; collagen and hyaline degenerated, and scar tissue replaced the main part of the tumor (Fig. 2). In cases of tumor recurrence, large amounts of tumor cells coexisted with radiation necrosis. Inflammation was observed at different stages after GKS; scattered phagocytic cells and lymphocytes surrounded the area of coagulation necrosis.

At 2-3 months after GKS, tumor vascular degeneration was evidenced by endothelial degeneration and fibrinoid necrosis, which further led to hyalinization, thrombosis, and lumen stenosis and obliteration (Fig. 3). Several years after GKS, cytoplasm vacuole degeneration, karyopyknosis, and abnormal morphology of the tumor cells adjacent to occluded vessels were observed. For several patients, histopathological examination revealed cavernous hemangioma-like vascular structures. Of note, no vessel wall hyalinization, fibrinoid necrosis, or vascular occlusion was observed in any pituitary adenoma specimens, even up to 10 years after GKS (Fig. 4). Further electron microscopy examination identified vascular basement membrane thickening in 1 patient with pituitary adenoma who had undergone surgery at 18 months after GKS.
Secondary pathological tumor changes, such as microhemorrhage, microcystic degeneration, myxoid degeneration, foam cell formation, and calcification, could be found, depending on the type of tumor. Microbleeding and hemosiderin deposition was obvious in more than half of the schwannomas. Microcystic degeneration was observed in all tumor types.

Among the 61 patients, 21 had undergone tumor resection before and after GKS, and specimens were collected before and after GKS for 9 patients. The interval between the first surgery and GKS was 3.5-120 months (median 9 months), and the interval between GKS and the final surgery was 8-144 months (median 46 months). Pathological examinations showed no increased tumor cell mitosis after GKS as compared with before GKS (Table 3).

\section{Discussion}

Conventional radiotherapy is rarely used to treat benign intracranial tumors. The Gamma Knife has a unique design that enables it to destroy the intracranial target area with a focused single high dose of radiation. The steep dose gradient makes possible radiosurgery that spares the surrounding normal tissue and preserves neuronal function. Most benign intracranial tumors belong to radiosurgery target category 2 (late-responding target surrounded by late-responding normal tissue). ${ }^{6}$ Tumors are well demarcated and not intertwined with normal brain tissue, which enables delivery of a high dose of radiation. After over 40 years of GKS use in clinical practice, there is more and more evidence-based data that support both the safety and the efficacy of stereotactic radiosurgery (SRS) treatment for benign intracranial tumors. ${ }^{2,11,13-15}$ The development of modern neuroimaging techniques and radiosurgery apparatus greatly improved radiation accuracy and enabled its application with various tumors. Therefore, SRS has become an indispensable neurosurgical technology.

In the study reported here, we evaluated 61 cases of benign intracranial tumors in which GKS followed by resection. We analyzed the reasons for surgical intervention, neuroimaging features, and pathological characteristics of different tumors at different times after GKS. The purpose of this study was to determine the pathological responses of benign intracranial tumors after a single high dose of radiation and, hence, to provide further insight for future clinical application.

TABLE 2: Interval between GKS and craniotomy, by tumor type

\begin{tabular}{lccccccc}
\hline & \multicolumn{7}{c}{ Interval Btwn GKS \& Surgery (mos) } \\
\cline { 2 - 8 } \multicolumn{1}{c}{ Tumor Type } & $\leq 6$ & $7-12$ & $13-24$ & $25-48$ & $49-60$ & $61-120$ & $>120$ \\
\hline meningioma & 4 & 4 & 4 & 2 & 1 & 6 & 3 \\
schwannoma & 2 & 1 & 4 & 9 & & 2 & \\
pituitary adenoma & 3 & 2 & 5 & 2 & & 2 & \\
hemangioblastoma & & & 2 & & & & \\
craniopharyngioma & & & 1 & 1 & & & \\
\hline
\end{tabular}


A. Liu et al.
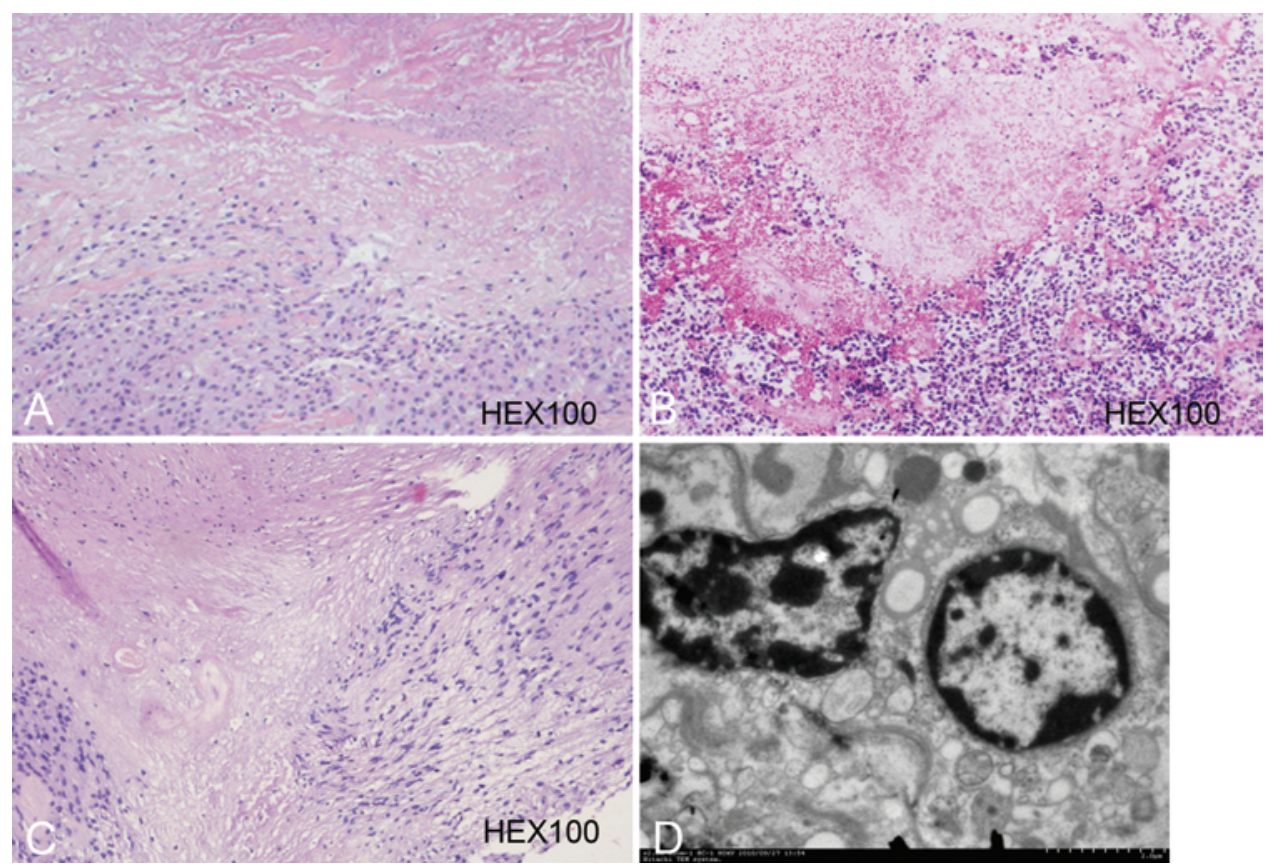

FIG. 1. Histological changes for different tumor types several months after GKS, showing coagulation necrosis with sharp demarcation in the tumor parenchyma. A: Meningioma 3 months after GKS. B: Pituitary adenoma 4 months after GKS. C: Schwannoma 3 months after GKS.

D: Schwannoma showing some apoptotic cells under the electron microscope.

\section{Clinical Analysis of Reasons for Surgical Intervention After} GKS

Tumor volume affects GKS effectiveness. In this study, most resections after GKS were performed because of large original tumor volume, increased tumor volume, and/or worsening of clinical signs and symptoms after GKS. Because of their harder and tougher texture, meningiomas undergo far less shrinkage after SRS than do schwannomas and pituitary adenomas. ${ }^{9}$ Thus, GKS should not be considered as the primary intervention for cases of extensive tumor mass effect.

Four patients in this group underwent open surgery despite tumor shrinkage after GKS: 1 with meningioma and cyst formation around the tumor and mass effect at 9
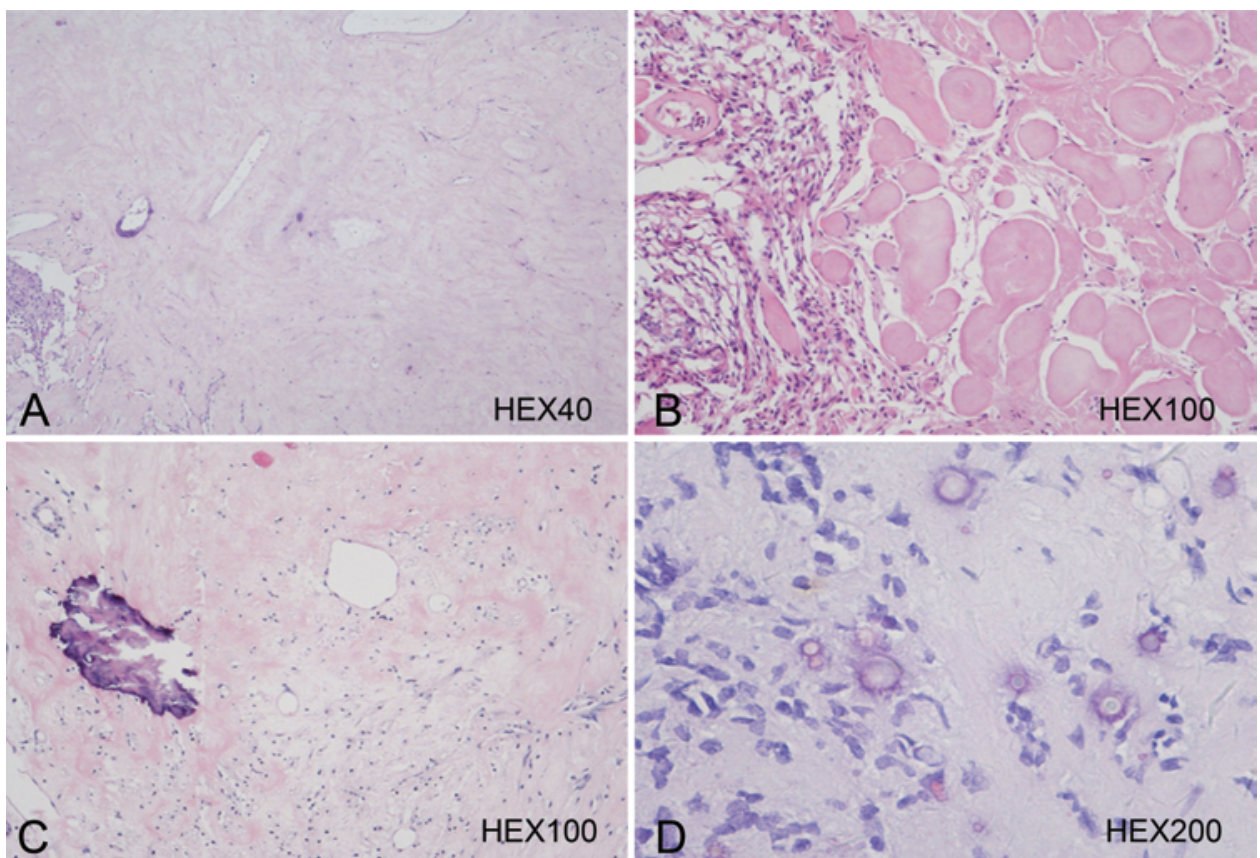

FIG. 2. Tumor parenchyma changes after GKS, showing collagenous degeneration; hyaline degenerated and scar tissue gradually formed. A: Meningioma (14 years after GKS). adenoma (10 years).

B: Meningioma (8 years). C: Schwannoma (4 years).

D: Pituitary 


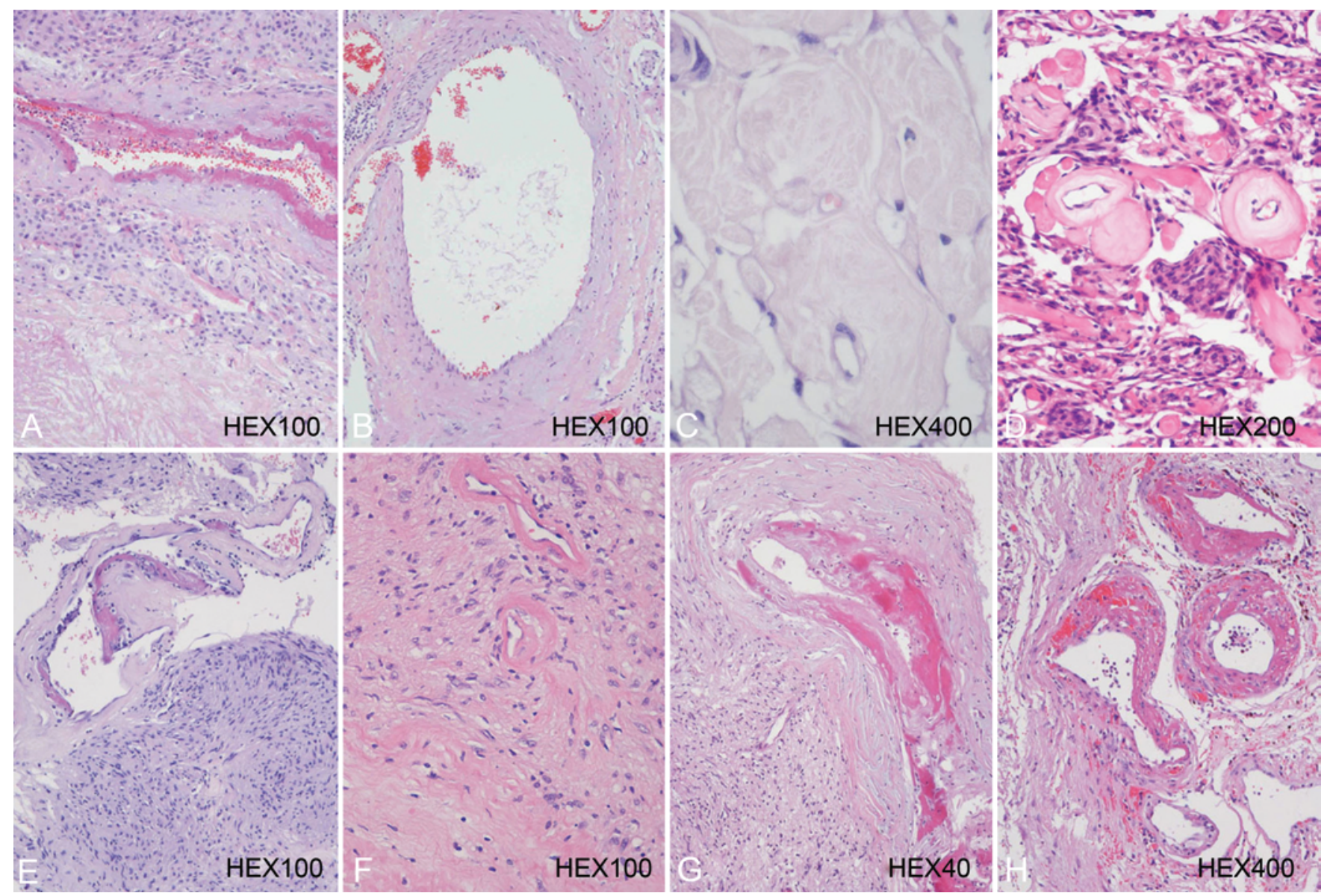

FIG. 3. Proliferative vasculopathy in the stroma after GKS, showing vascular changes of meningiomas at different intervals (A-D). Endothelial destruction at 3 months (A), fibrinoid necrosis in the vessel wall at 8 months (B), and lumen obliteration at 5 years (C) and 9 years (D) after GKS. E-H: Schwannomas. Vascular degeneration with thrombosis at 11 months (E), collagen degeneration of vessel wall with lumen stenosis at 2 years $(\mathbf{F})$, and the lumens nearly occluded at 3 years $(\mathbf{G})$ and totally occluded at 4 years $(\mathbf{H})$ after GKS.

years after GKS (Fig. 5A-C), 1 with trigeminal schwannoma and unbearable trigeminal neuralgia at 32 months after GKS, and 2 with acoustic tumor and gradual enlargement of supratentorial ventricle at 24-32 months after GKS. We predict that hydrocephalous might be caused by a change in CSF composition. For such cases, ventriculoperitoneal shunting or a ventriculostomy of the third ventricle might help relieve the symptoms of hydrocephalus.

Of the 14 patients with pituitary adenoma, most $(n=$ 10) underwent surgical intervention because of tumoral hemorrhage. All 10 of these patients experienced acute or subacute bleeding and increased tumor volume, which led to headache and vision dysfunction. Immediate surgical intervention for decompression and vision protection were needed. The 9 patients who received GKS at our hospital accounted for only $0.3 \%$ of all pituitary adenoma cases treated by GKS during the same period; this rate is lower than the pituitary apoplexy rate of $1.6 \%-12.8 \% .^{12}$ Therefore, it is unlikely that GKS increases the rate of pituitary adenoma hemorrhage.

\section{Treatment Planning Analysis}

Previous studies have indicated that a margin dose of 12-16 Gy could achieve long-term tumor control.,3,4,17 However, comprehensive dose distribution over the whole tumor is critical for preventing delayed recurrence. During the 1990s, to prevent tumor growth we prescribed a high dose to the attachment area of the petrous ridge in 1 patient with a large cerebellopontine angle meningioma. At 10 months after GKS, the patient underwent tumor resection because of symptomatic intracranial hypertension. The tumor was white in the irradiated region because of lack of blood supply; normal blood supply was found in the nonirradiated area. For the residual tumor after resection, especially for meningiomas and pituitary adenomas, the dose distribution should cover all tumor area; comparing neuroimages before craniotomy is very helpful for dose planning.

\section{Tumor Pathology Analysis}

During the 1960s, focused gamma irradiation at a dose of more than 200 Gy was used for thalamotomy to treat intractable pain. Autopsies conducted a few months after radiation identified a well-demarcated radiation lesion of coagulation necrosis surrounded by astrogliosis. Normal brain structure was preserved outside the target- 
A. Liu et al.

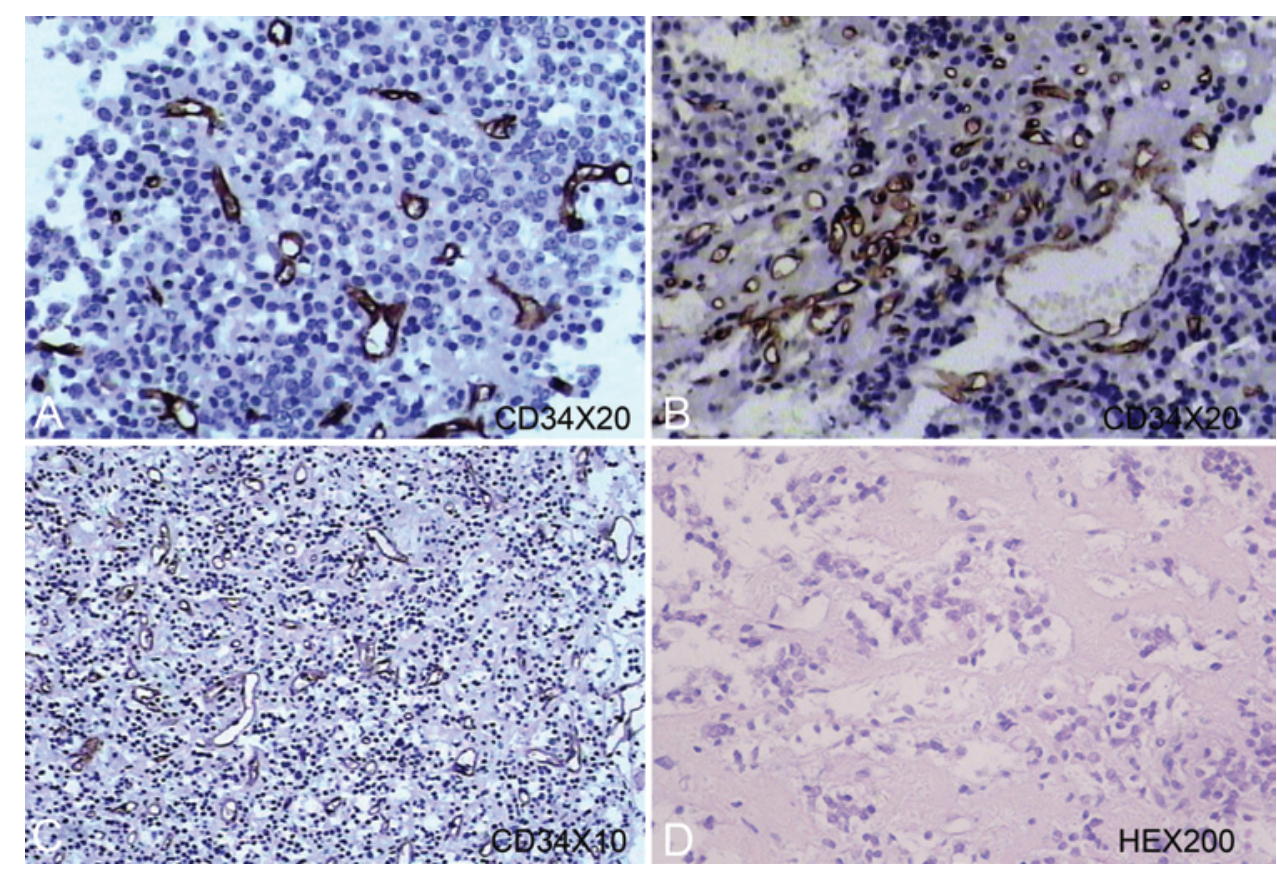

FIG. 4. Vessel changes in pituitary adenoma at 6 (A), 22 (B), 38 (C), and 120 (D) months after GKS, showing many thin-walled vessels almost with normal morphology. The proliferation of the vessel walls was hard to find even several years after irradiation.

ed area. Radiation reaction was also found in blood vessels and included vessel wall intimal thickening, fibroid necrosis, vascular thrombosis, and stenosis. ${ }^{7,8}$ One study demonstrated that a dose of at least 140 Gy is needed to form an obvious radiosurgical lesion in the human brain. ${ }^{16}$ These studies have established the foundation for the clinical application of radiosurgery.

The 3 main morphological types of radiation-induced tissue reactions have been recognized as acute, subacute, and chronic. ${ }^{2}, 18$ An acute histological reaction may develop early or during the delayed period, but a chronic tissue response evolves only during the delayed stage. Our findings are consistent with those of previous studies. The reaction to radiosurgery is a continuum of pathological changes. All specimens obtained a few months after GKS showed coagulation necrosis, scattered apoptosis, interstitial edema, and reactive astrogliosis, which could contribute to the transient exacerbation of clinical symptoms in some patients. In a few patients, severe damage to normal brain tissue around the tumor, caused by the equipment and technical issues, has been found. At the late stage, coagulation necrosis is replaced by scar tissue, which corresponds to the shrinkage of tumor volume observed in neuroimaging scans. The degree of tumor shrinkage depends on the texture of different tumors. For example, tumors with more microcystic degeneration, interstitial fluid, myxoid matrix, and soft texture usually shrink more significantly after SRS. Inflammatory reaction around the

TABLE 3: Comparison of WHO classification of tumors of the CNS and mitotic counts for pathological specimens collected before and after GKS*

\begin{tabular}{|c|c|c|c|c|c|c|c|c|}
\hline \multirow[b]{2}{*}{ Case No. } & \multirow[b]{2}{*}{ Age (yrs), Sex } & \multirow[b]{2}{*}{ Diagnosis } & \multirow[b]{2}{*}{ Op-GKS (mos) } & \multirow[b]{2}{*}{ GKS-Op (mos) } & \multicolumn{2}{|c|}{ WHO Classification } & \multicolumn{2}{|c|}{ No. of Mitoses/10 hpf† } \\
\hline & & & & & Before GKS & After GKS & Before GKS & After GKS \\
\hline 1 & $55, \mathrm{M}$ & meningioma & 6 & 111 & I & 1 & 0.4 & 0.2 \\
\hline 3 & $57, \mathrm{~F}$ & meningioma & 5 & 9 & I & 1 & 1.8 & 1 \\
\hline 4 & $36, F$ & meningioma & 9 & 144 & I & I & 0.6 & 0.6 \\
\hline 7 & $17, \mathrm{M}$ & pituitary adenoma & 3.5 & 8 & ‡ & I & 0.6 & 0.2 \\
\hline 8 & $43, F$ & pituitary adenoma & 24 & 18 & १ & ‡ & 0.2 & 0.4 \\
\hline 9 & $60, M$ & craniopharyngioma & 48 & 46 & け & ‡ & 1 & 1.2 \\
\hline
\end{tabular}

* GKS-Op = interval between GKS and final surgery; Op-GKS = interval between the first surgery and GKS.

$\dagger$ Number of mitoses $/ 10 \mathrm{hpf}=$ number of mitoses in at least $50 \mathrm{hpf}$ divided by 10 .

$\ddagger$ Score is the equivalent to that of WHO Grade I. 


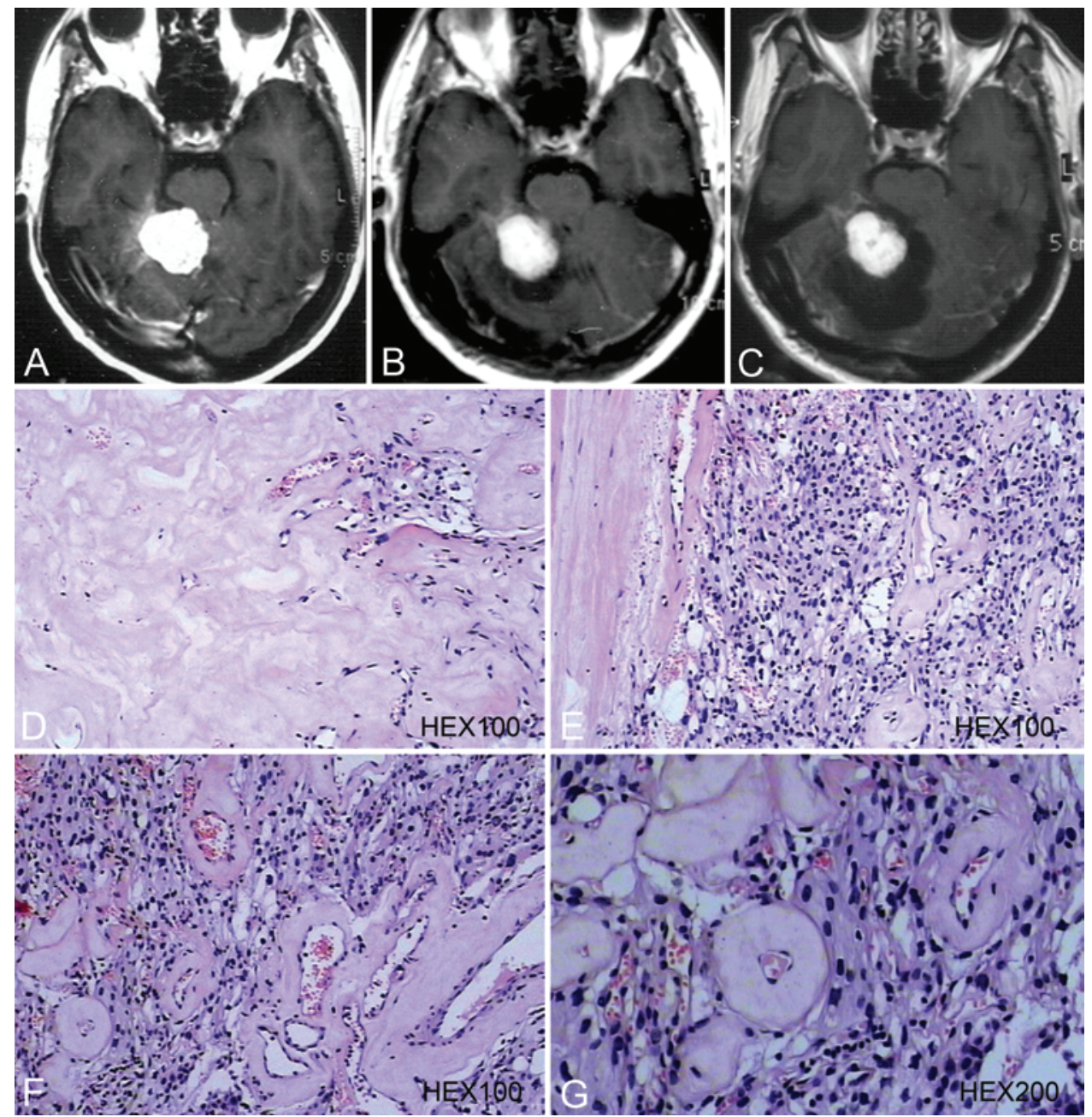

FIG. 5. A-C: MR images obtained in a 55-year-old man in whom the first open surgery for a right tentorial meningioma failed. The patient underwent GKS 2 times, 7 months apart ( 9 Gy and 40\%, 8 Gy and 40\%, respectively). Nine years later, the tumor had shrunk, but cyst formation caused mass effect and craniotomy was again performed. A: Before GKS. B: Five years after GKS. C: Nine years after GKS. Histopathological examination revealed that scar tissue and hyaline degeneration had replaced the parenchyma and stroma in the central part of the tumor (D), some tumor cells still alive with pyknotic cells in the target margin (E), and obliteration of most vessels ( $F$ and $G)$.

target, such as infiltration of phagocytic cells and lymphocytes, is part of the repair process, and the extent of inflammation depends on the interval after radiation and the vascularity of the tumor. The ultimate destruction of the tumor tissue is a chronic process that results from the radiation-induced damage, scar formation, and loss of blood supply caused by vessel stenosis and occlusion (Fig. 5), which could take several years. However, the residual tumor cells at the periphery of the target can grow and lead to tumor relapse after blood supply is established. In the study reported here, residual tumor cells were found at the periphery of the target in 4 patients, despite remarkable tumor shrinkage after GKS. Low margin dose and dose inhomogeneity in the targeting area might spare a small number of tumor cells, which could result in tumor relapse. Thus, long-term follow-up is necessary. Of note, vascular proliferation after SRS is similar to the atherosclerotic vascular changes that occur in patients with long-term hypertension; this similarity might partially explain the slower tumor growth that occurs in older pa- tients. In patients with pituitary adenoma, no vascular degeneration was observed after radiation. We speculate that the unique sinusoidal and thin-walled vessels might render them more resistant to stenosis. Further studies are warranted to determine whether the lesser vascular reaction contributes to the slow reduction of high hormone levels in functional adenomas after SRS.

Intratumoral hemorrhage and cystic degeneration are inherent pathological changes of brain tumors, and the extent varies among different types of tumor. The single high dose of radiation used with GKS not only kills tumor cells but also accelerates the tumor degeneration process. Tumor hemorrhage and cystic degeneration can be identified by neuroimaging and/or histological examination. Hemorrhage of pituitary adenomas can often be seen on the neuroimages; however, hemorrhage of schwannomas is mostly microbleeding, which can be hard to identify by conventional neuroimaging. Microbleeding (Fig. 6) is seen as punctate long T1, mixed T2, and spotted enhancement on gadolinium-DTPA (diethylenetriamine- 

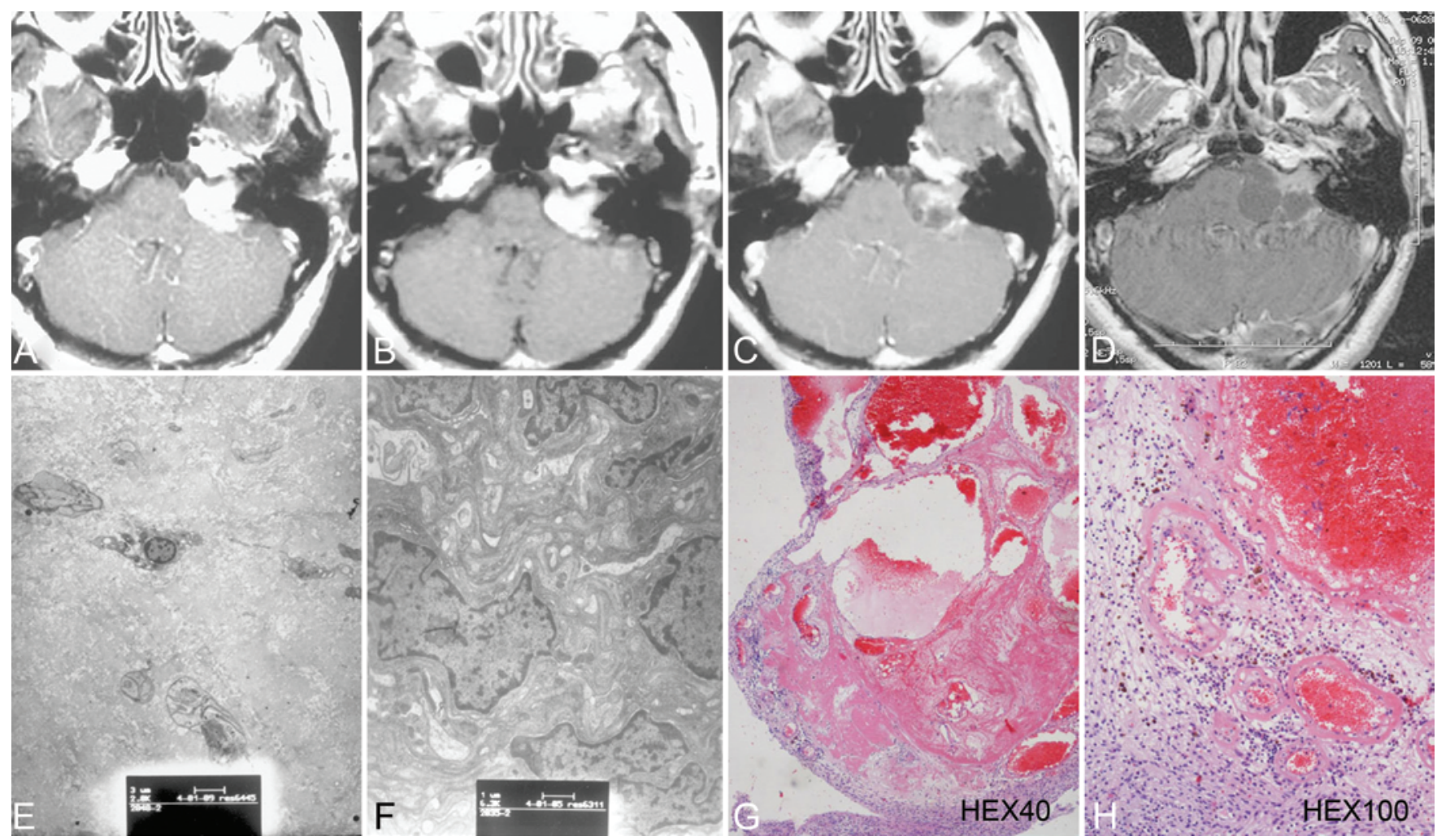

FIG. 6. Vestibular schwannoma. The patient underwent microsurgery 7.5 years after GKS because of cyst formation and compression of the brainstem. Serial Gd-enhanced axial MR images obtained before (A) and at 2 (B), 5 (C), and 7.5 (D) years after GKS. Histopathological examination revealed scar tissue in the center of the target (E) and nuclei deformation (F) under an electron microscope, and hyaline degeneration in the vessel wall $(\mathbf{G})$ and some microhemorrhage and hemosiderosis in the tumor $(\mathbf{H})$ under a light microscope.

pentaacetic acid) scans. Usually, MR images show loss of contrast enhancement in the center of acoustic schwannomas a few months after GKS, which predicts future tumor shrinkage. We found that tumor volume enlargement following the obvious loss of enhancement normally predicts microbleeding or hemosiderin deposition in histologic examination. Recurrent microhemorrhage can lead to cystic degeneration and increased schwannoma volume (Fig. 6). Surgical intervention is needed when symptoms are exacerbated. For meningiomas, with their rubbery and hard texture, cystic formation and hemorrhage are rarely found during neuroimaging examination, even if some microcystic degeneration can be observed under the microscope. We observed intratumoral hemorrhage by neuroimaging in 1 patient with petroclival meningioma at 26 months after GKS. Further histological examination demonstrated abnormal thin-walled vessels in the tumor.

In our study population, 7 patients with obvious cyst formation seen on neuroimages required surgical intervention, including all those with hemangioblastoma and craniopharyngioma. Hemangioblastomas are usually mixed solid and cystic tumors without a cyst wall. The cyst fluid of hemangioblastomas may be secreted by tumor nodules and contains components similar to plasma (i.e., higher protein level than CSF). Although SRS can induce radiation reactions in tumor nodules and control tumor growth, the cyst could expand because of the higher osmotic pressure of the cyst fluid. In contrast, the cyst wall of craniopharyngiomas is composed of tumor cells with a secretory function. The cyst fluid contains variable amounts of cholesterol crystals and keratinous debris. SRS cannot provide timely control of the fluid secretion. The cyst could expand within a few weeks or months after treatment, leading to further vision impairment, which requires immediate surgical intervention.

There has been concern whether SRS can increase the risk for malignant transformation of benign brain tumors. ${ }^{1}$ In the study reported here, no increased tumor cell mitosis after GKS was found. Heterogeneity of the tumor cells could render different growth rates in the same type of benign tumor. For meningiomas, part of the tumor might demonstrate extensive invasion and active mitosis despite its overall benign behavior. In our recent study, we found that the major reason for meningioma resection after failed GKS was the presence of atypical and active proliferative meningioma cells. ${ }^{10}$ In fact, patients with recurrent meningioma after SRS normally underwent resection before radiation, some even more than once. Both surgical intervention ${ }^{5}$ and Gamma Knife radiation could act as physical stimuli that change the metabolism of the tumor and surrounding brain tissue. The primary and secondary actions of GKS can last years, even decades. Both favorable and unfavorable effects of SRS on benign brain tumors should be considered. 


\section{Conclusions}

Our findings indicate that GKS is an effective intervention for small, deep, recurrent, or residual benign brain tumors. However, GKS should not be considered as the first choice if rapid decompression is needed for the mass effect of benign brain tumors. In this study, the histopathological changes were observed for a small group of patients for whom GKS failed and, thus, may not be representative of typical changes seen in patients for whom GKS was successful. Nonetheless, for these patients, single high-dose radiation induces biological effects on tumor cells and vasculature. We found that SRS did not promote tumor proliferation while accelerating the degenerative process of the benign tumor. Primary and secondary effects of gamma irradiation to the tissues can last up to decades. Long-term clinical follow-up is necessary for patients who have undergone GKS.

\section{Acknowledgment}

We thank Shao-Hua Yang, M.D., Ph.D., Professor and Vice Chair, Department of Pharmacology and Neuroscience, University of North Texas Health Science Center, for his assistance with editing of this article and consistent support for our research work.

\section{Disclosure}

The authors report no conflict of interest concerning the materials or methods used in this study or the findings specified in this paper. The study was partially support by grants from The Capital Health Development Projects (no. CHDP-2011-1015-02).

Author contributions to the study and manuscript preparation include the following. Conception and design: Liu, JM Wang. Acquisition of data: all authors. Analysis and interpretation of data: Liu, JM Wang, Li, YL Sun. Drafting the article: Liu. Critically revising the article: Liu, JM Wang. Reviewed submitted version of manuscript: Liu, JM Wang. Approved the final version of the manuscript on behalf of all authors: Liu. Statistical analysis: Liu, JM Wang. Administrative/technical/material support: Liu, JM Wang, YL Sun, SB Sun, Luo, MH Wang. Study supervision: Liu.

\section{References}

1. Akamatsu Y, Murakami K, Watanabe M, Jokura H, Tominaga T: Malignant peripheral nerve sheath tumor arising from benign vestibular schwannoma treated by gamma knife radiosurgery after two previous surgeries: a case report with surgical and pathological observations. World Neurosurg 73: 751-754, 2010

2. Fajardo LF, Berthrong M, Anderson RE (eds): Radiation Pathology. New York: Oxford University Press, 2001

3. Flickinger JC, Kondziolka D, Niranjan A, Maitz A, Voynov G, Lunsford LD: Acoustic neuroma radiosurgery with marginal tumor doses of 12 to 13 Gy. Int J Radiat Oncol Biol Phys 60:225-230, 2004

4. Hasegawa T, Kida Y, Kato T, Iizuka H, Kuramitsu S, Yamamoto T: Long-term safety and efficacy of stereotactic radiosurgery for vestibular schwannomas: evaluation of $440 \mathrm{pa}-$ tients more than 10 years after treatment with Gamma Knife surgery. Clinical article. J Neurosurg 118:557-565, 2013
5. Krayenbühl N, Pravdenkova S, Al-Mefty O: De novo versus transformed atypical and anaplastic meningiomas: comparisons of clinical course, cytogenetics, cytokinetics, and outcome. Neurosurgery 61:495-504, 2007

6. Larson DA: Radiosurgery and fractionation, in Kondzioka D (ed): Radiosurgery 1995. Basel: Karger, 1996, Vol 1, pp 261-267

7. Larsson B, Leksell L, Rexed B: The use of high energy protons for cerebral surgery in man. Acta Chir Scand 125:1-7, 1963

8. Leksell L: Cerebral radiosurgery. I. Gammathalanotomy in two cases of intractable pain. Acta Chir Scand 134:585-595, 1968

9. Liu AL, Wang C, Sun S, Wang M, Liu P: Gamma knife radiosurgery for tumors involving the cavernous sinus. Stereotact Funct Neurosurg 83:45-51, 2005

10. Liu AL, Wang JM, Li GL, Sun YL, Luo B, Sun SB, et al: [The analysis of craniotomy and pathology of meningiomas after failed Gamma knife radiosurgery.] Chin J Neurosurg 29:435-440, 2013 (Chinese)

11. Maniakas A, Saliba I: Microsurgery versus stereotactic radiation for small vestibular schwannomas: a meta-analysis of patients with more than 5 years' follow-up. Otol Neurotol 33:1611-1620, 2012

12. Möller-Goede DL, Brändle M, Landau K, Bernays RL, Schmid C: Pituitary apoplexy: re-evaluation of risk factors for bleeding into pituitary adenomas and impact on outcome. Eur J Endocrinol 164:37-43, 2011

13. Norén G: Long-term complications following gamma knife radiosurgery of vestibular schwannomas. Stereotact Funct Neurosurg 70 (Suppl 1):65-73, 1998

14. Santacroce A, Walier M, Régis J, Liščák R, Motti E, Lindquist $\mathrm{C}$, et al: Long-term tumor control of benign intracranial meningiomas after radiosurgery in a series of 4565 patients. Neurosurgery 70:32-39, 2012

15. Sheehan JP, Starke RM, Mathieu D, Young B, Sneed PK, Chiang VL, et al: Gamma Knife radiosurgery for the management of nonfunctioning pituitary adenomas: a multicenter study. Clinical article. J Neurosurg 119:446-456, 2013

16. Steiner L, Forster D, Leksell L, Meyerson BA, Boëthius J: Gammathalamotomy in intractable pain. Acta Neurochir (Wien) 52:173-184, 1980

17. Sun S, Liu A: Long-term follow-up studies of Gamma Knife surgery with a low margin dose for vestibular schwannoma. Clinical article. J Neurosurg 117 Suppl:57-62, 2012

18. Szeifert GT, Kondziolka D, Atteberry DS, Salmon I, Levivier M, Lunsford LD: Radiosurgical pathology of brain tumors: metastases, schwannomas, meningiomas, astrocytomas, hemangioblastomas. Prog Neurol Surg 20:91-105, 2007

Manuscript submitted June 28, 2014.

Accepted August 4, 2014.

Presented in abstract form at the 17th International Leksell Gamma Knife Society Meeting, New York, New York, May 11-15, 2014.

Please include this information when citing this paper: DOI: 10.3171/2014.8.GKS141439.

Address correspondence to: Ali Liu, M.D., Gamma Knife Center, Beijing Neurosurgical Institute, No. 6, Tiantan Xili, Beijing 100050, China.email: aliliu3@sina.com. 